\title{
Customer facing components for network management systems
}

\author{
I. Busse, S. Covaci \\ GMD FOKUS, Hardenbergplatz 2, 10623 Berlin, Germany \\ Phone: +49 3025499 179, Fax: +49 3025499 202, Email: busse@fokus.gmd.de
}

\begin{abstract}
The paper discusses the use of the WWW and Java in the provisioning of broadband connectivity to a big number of customers by a public network operator. Several options are analysed, ranging from a simple interface for service activation and usage to an integrated solution including outsourcing of customer network management by means of components dynamically loaded to the customer site. Besides the quick access to the service provided by the WWW, the paper describes the additional benefits drawn from the portability and extensibility of Java based solutions. Features like management functionality on demand, automatic release and update management of software, reduction in network load by moving functionality close to the data source are discussed in detail. The paper introduces an appealing alternative or complement to any network management solution. A prototype implementation currently under development is used for the validation of the proposed solutions in an intranet environment.
\end{abstract}

\section{Keywords}

Broadband connectivity, network management, service management, outsourcing, mobile agents, java

\section{MOTIVATION}

Public network operators interconnect many customer premises networks via their broadband wide-area networks. While most of the customers already have network management solutions in operation to configure and monitor the state of their local networks they seldomly, if at all, get insight into public domains. Within public domains it is still very common that the setup and trouble shooting of connections is done by means of telephony and fax. This is a weak point for companies with distributed sites like for example distributed production plants that rely on wide-area connections.

A network operator is interested in providing his services with a minimum of human involvement in order to keep the cost down and to avoid human errors. Using network management solutions he can increase the availability of his network. The management solution can be offered to the customer or the provider can take over the task of network management also within the customer domain. 
The customer wants to get an easy to use, reliable service at reasonable cost. This can only be achieved with an integrated network management solution including the national and international connections between the customer premises networks.

The following network management operations should be offered by a public network operator to the customers of his broadband connectivity service:

- subscription

- reservation, activation, modification, and deactivation of connections (if not done by inband signalling)

- testing of connections (prior to and during usage)

- receiving alarms

- trouble ticketing

- performance monitoring

- accounting.

There are several organizations working on interface specifications for this functionality:

Within the Internet community SNMP based specifications are developed [RFC1695]. The ATM Forum has defined management links between public and private networks in a protocol independent manner [M3] and adopted the Internet mib as a mapping to SNMP. They focus on the network management level, i.e., only connection setup, performance monitoring, and fault alarms are covered. There are initial proposals for an extension to the service management level, e.g., the exchange of accounting information [ACCT96].

There are other initiatives also studying the customer-to-provider reference point, like the Network Management Forum and the Eurescom ${ }^{1}$ projects. The Network Management Forum solution sets take into account the whole business process. The actual interface specification are provided in CMIP. The Eurescom work includes the service management level but is weak on the network management level that is currently restricted to virtual path configuration and alarm surveillance.

All the above mentioned work put the emphasize on the provision of a standardized management interface. Currently they use as management protocol SNMP and CMIP, and in the future Corba is considered an alternative.

For several reasons the provision of just a management protocol-based interface seems to be insufficient:

1. It is likely that the first contact to a connectivity provider will be made via a medium like the WWW. Here the potential customer can browse and compare offerings or let a broker facility select one according to its criteria. This is especially of interest if the connectivity provider is a player that is distinct from the network access provider, so that a customer is free to choose anyone according to his own preferences, e.g., the tariff.

2. Many customers, especially small companies, may not have management platforms installed and therefore no access to low level management protocol interfaces. In such cases it is useful to provide a ready to use graphical user interface for at least configuration and monitoring purposes. Also a help desk facility for the customer seems to be a necessary basic component.

3. Even if a management platform is available there is usually no support for the service management level. While there is a lot of off-the-shelf functionality to gather

1. Eurescom is an initiative of the European network operators. 
network performance data and to collect and correlate alarms to isolate faults, the service management level functionality can hardly be used. So even for companies with installed network management solutions additional plug-ins or standalone solutions are necessary to enable access to the full functionality of the connectivity service.

4. If the connectivity service provider also offers to do the network management in the customer domain, additional functionality is required at the customer-to-service provider interface. The service provider must be able to install network management components at the customer site and enable an information flow for the network management level information in the reverse direction while a service management level information exchange still takes place as mentioned before. This scenario is often referred to as outsourcing of the network management activities.

5. Normally all software is updated sooner or later, thus requiring software distribution mechanisms for any kind of software that is downloaded and stored within the customer domain. This kind of functionality is currently not addressed at the considered management interfaces.

As we can see solutions (see Figure 1) range from a simple customer interface (a) and components for the integration into own legacy management platforms (b) to outsourcing of the network management (c).

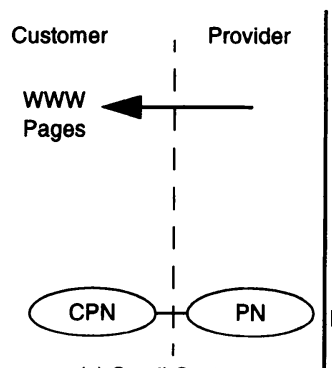

(a) Small Customer Solution

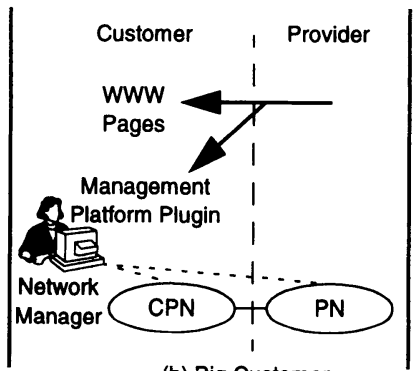

(b) Big Customer Solution

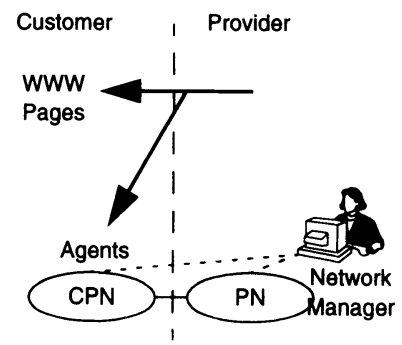

(c) Outsourcing Solution

CPN: Customer Premises Network PN: Public Network

Figure 1 Customer facing network management components.

In the subsequent sections we will show the usability of a WWW interface and of Java based mobile agents for the purpose of provisioning and management of an end-to-end connectivity service. The second section discusses a simple WWW based interface to access the broadband connectivity management. The third section describes the benefits we can draw from mobile agents in such an environment. Then we describe how agents can easily be implemented based on Java. The fifth section presents an example scenarios that we are currently prototyping. The paper is closed by describing the relation to other work in the area of client-server computing and mobile agents, and a final summary. 


\section{WWW BASED CUSTOMER-TO-SERVICE PROVIDER INTERFACE}

As already indicated above the first contact with potential customers might take place via the WWW. A connectivity provider should be present with an appropriate service offering. To allow for comparison of competitive service providers, the service characteristics, e.g., pricing information, should be available and ready to use for brokerage services. This information is more or less static and can be retrieved using http from a freely accessible WWW server.

As the next step a subscription is required. It settles a contract between the customer and the provider and should enable the provider to bill its customer. Due to security issues this step still can't be done electronically. Nevertheless for the operation of the service some information, e.g., addressing information, has to be available in electronic form. This information can be entered by the customer and/or by a service administrator in the provider domain. The final goal should be to fully automate this procedure as soon as the security facilities and the legal base are available.

After a contract is "signed" the customer gains access to the relevant software components. During the contract negotiation or during the subsequent installation phase the necessary service components can be selected by the customer. The customer will be billed also according to the chosen components.

A simple application for the connectivity provisioning includes just a user interface for connection configuration. Additional packages may allow for alarm surveillance or performance monitoring. Since a customer might not have a legacy management platform we propose to offer an interface based on the WWW for this purpose. A prototype user interface is shown in the following Figure 2. All components share a common user interface as soon as they are installed by the customer.

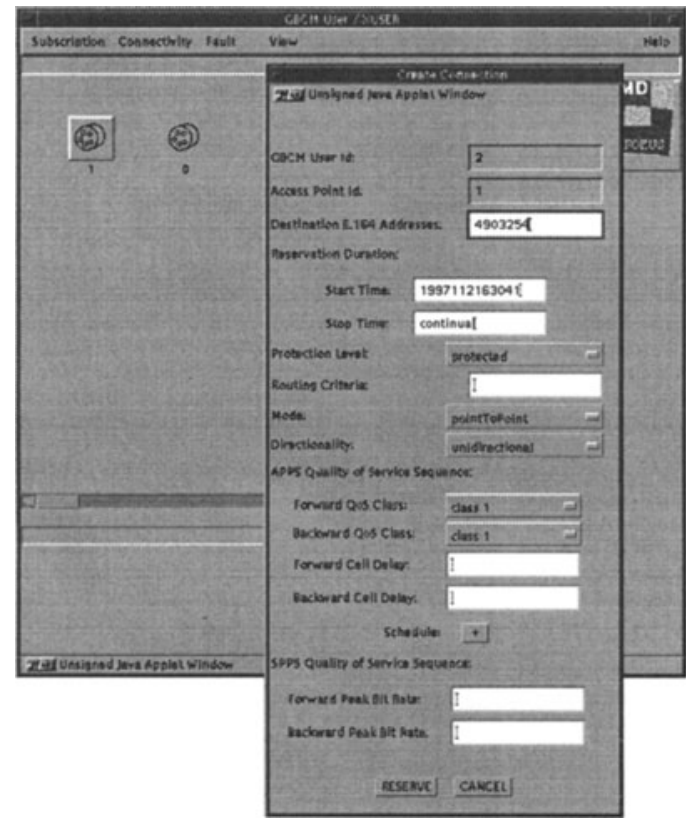

Figure 2 Prototype user interface. 
There are several options for communicating with the service provider domain. Due to the static nature of http in that it is hard to code state information this is implemented as a Java applet or application. The applet communicates with a dedicated server in the service provider domain (Figure 3).

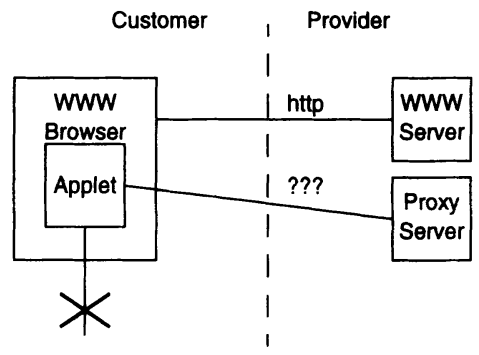

Figure 3 Architecture and communication protocol options between customer and service provider.

At the customer-to-service provider reference point we can use the standardized interfaces based on SNMP/ CMIP, a private socket interface or Corba. To get a light solution it seems to be reasonable to settle on code that is included in the core Java distribution and is therefore already present at the customer site. SNMP and CMIP require special protocol implementations that have to be downloaded with the user interface since they are not included in the class libraries of the wide-spread browsers. For SNMP there are already two implementations, see [ADVENT] and [JASCA], that might be used. CMIP does not seem to be feasible at all due to its complexity.

A socket can be easily driven from within an applet and therefore provides a ready to use solution to any information exchange with the service provider domain. We decided to take a private socket based interface that carries the commands invoked at the user interface. This greatly reduces the required code at the customer site, leaving the mapping onto the necessary management interactions to the server inside the service provider domain. A number of platform providers like e.g. IBM and HP offer a string based API that can also very easily be used for this purpose [DERI]. With such an approach we might benefit from a standardized and thus portable string API to SNMP and CMIP.

For the future Corba [CORBA] and Java Remote Method Invocation [JRMI] are of interest since both will become part of the core Java and will thus be available in all Java enabled browsers. There is an increasing community promoting Corba in the standardization of service interfaces including the area of network management. The interfaces are developed separately or derived from existing mib specifications according to the SNMP SMI [RFC1157] or GDMO [ISO10165-4], making use of the mapping defined by the X/Open JIDM working group [XOJIDM]. Nevertheless, the Corba environment still lacks the management facilities provided by SNMP or CMIP agents. CMIS allows to perform operations on many objects or attributes specified e.g. by the means of scoping and filtering.

To keep applets from spying networks behind firewalls many browsers limit the destinations for socket set-up to the host from which an applet was loaded. Therefore the usability of the browser for the purpose of network management is poor since an applet cannot connect to an arbitrary network or network element manager. A workaround might be a proxy server for management requests within the provider domain. 
In any of the afore mentioned solutions it is useful for such an implementation to be stored locally. This limits the number of downloads to the time of the subscription and the subsequent changes of the software configuration. Also service specific functions like logging of configuration requests and faults can be implemented more easily with the ability to save state information to the local disk. Unfortunately, the security restrictions of the available browsers do not allow any local disk access. It is desirable that a user of a browser can configure at least a single directory for being accessed by the downloaded applets.

An overview of the security restrictions is given in the following table. Note, that it makes a difference whether an applet is loaded over the network or from the local disk. Of course, the basic Java Virtual Machine (JavaVM) grants all permissions to an application.

Table 1 Overview of security restrictions

\begin{tabular}{llllll}
\hline & \multicolumn{2}{l}{ Netscape Navigator } & \multicolumn{2}{l}{ AppletViewer } & JavaVM \\
\cline { 2 - 5 } & net & local & net & local & \\
\hline file access & no & no & $\begin{array}{l}\text { access } \\
\text { control list }\end{array}$ & yes & yes \\
$\begin{array}{l}\text { socket } \\
\text { to any host }\end{array}$ & no & yes & no & yes & yes \\
\hline
\end{tabular}

\section{EXTENSIBLE AND MOBILE AGENTS - WHY?}

An even more interesting approach is the possibility of using Java for the implementation of management agents. This allows to extend the functionality of an agent on demand. A further step would be to have mobile agents. Just a JavaVM needs to be in place. If a specific management task has to be fulfilled a roaming agent is sent around. It can provide interfaces supporting all protocols that are fully implemented in Java. Note, that a browser is not sufficient for this task, since agents should be able to do processing even if the user interface is not running.

There are several benefits we can draw from this approach:

1. In principle, there is no need for any running management agent if there is no manager that requires a specific information. As such the computing and communication overhead can be reduced by putting the agent first in place when a specific management action has to be performed and to destroy it when there is no longer a need for it.

2. Certain management tasks, like gathering usage data and computing charges, for example, can be located close to the real resources. Then we have only the reduced accounting data that need to be transported. This would reduce the communication overhead and thus the network load.

3. Sooner or later each piece of software is outdated, e.g., because a bug was discovered and fixed or because a new environment requires a different or extended functionality. An agent or the functionality within an agent can be replaced by a new 
version of the software from remote. This is a basic functionality available in an agent execution environment.

4. The ability to move functionality to several places for execution allows for load balancing and results in a fault tolerant system. Agents can be directed to places with free computing power. In case an agent fails the functionality can be loaded in a different place on another host. Each host running the agent execution environment can act as such a back-up system.

5. A provider can react flexible on the changing environment in a customer domain within the already mentioned outsourcing scenario. A roaming agent can collect configuration data about the customer domain and return to the provider. Further agents are configured and sent to the customer domain according to the equipment installed and reported by the spy, and the management functionality required without an interaction between the customer and the provider. In case of special fault conditions additional agents for fault analysis can be sent to the customer domain performing analysis tasks.

\section{EXTENSIBLE AND MOBILE AGENTS - HOW?}

A suitable environment for extensible and mobile agents requires an execution environment that is available on a number of platforms. Code and state information needs to be portable from one execution environment to the next. Java seems to provide a good base for this purpose.

The JavaVM is available on all common computing platforms and provides a machine independent execution environment. It was designed for downloading and executing code from WWW servers therefore the basic functionality for moving code is available. A first step for moving the state of objects is provided by the object serialization facility [JOS] currently released by Sun. It allows to stream an object including all referenced objects with minimum effort. Based on object serialization the remote method invocation facility [JRMI] allows for passing arbitrary objects from JavaVM to JavaVM between two networked hosts.

Our agent execution environment has four basic components: agencies, agents, a name server and a class server. In the following we will roughly describe the purpose of each component.

\subsection{Agency}

An agency has three basic objects: a factory, a context and a security manager. The factory provides a remote interface. The method createAgent(Agent) implements a generic agent factory that is needed to create arbitrary agents from remote. Each factory can be uniquely identified with the hostname and the port number. It is registered at the central name server.

When an agent is created its object reference is stored within the context object in order to keep track of all agents at an agency. Then the agent is started within a separate thread executing the method run() of the agent object. Such an agent factory at each place provides the minimum infrastructure for mobile agents.

The security manager object will be based on the security feature of JDK 1.1 and is not yet fully implemented. 


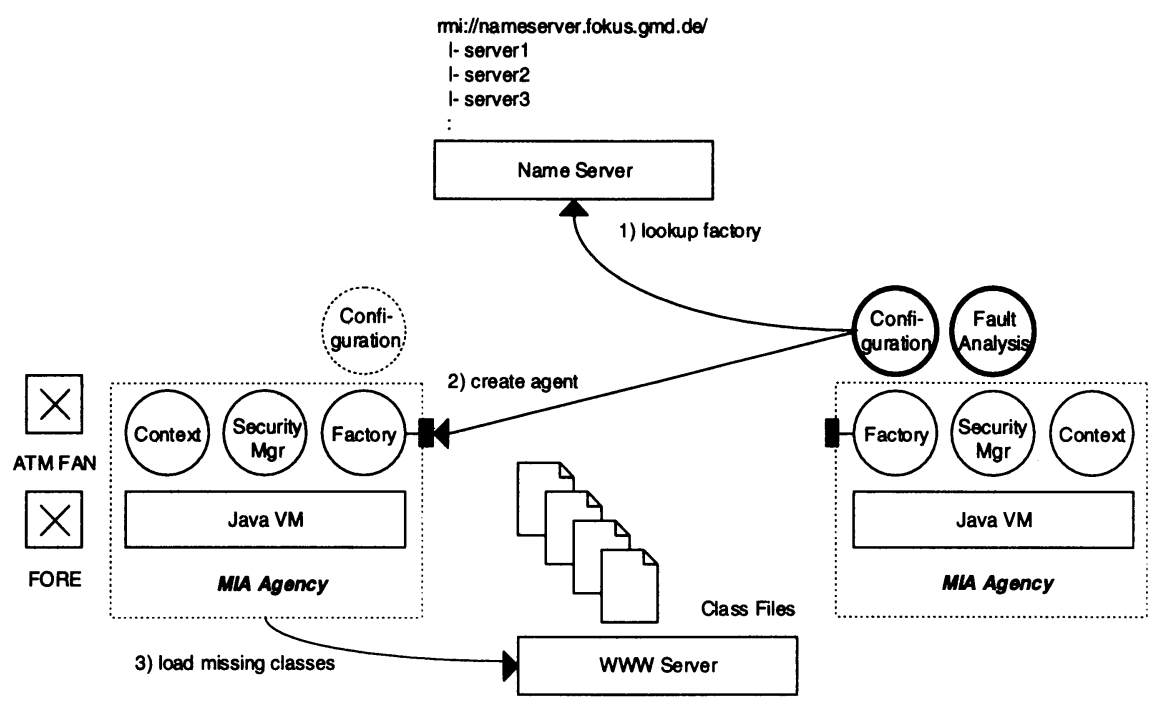

Figure 4 Mobile intelligent agent (MIA) execution environment.

\subsection{Agent}

The class Agent implements the behavior that is common to all agents. It is abstract and cannot be instantiated. Within a subclass the specific behavior needs to be implemented first.

An agent has two basic attributes: agent identifier, that is globally unique, and classbase, to locate and load missing code over the network. Note, that there might be code missing even after the agent ran for many hours and changed its place several times. So from any place where code is detected to be missing the execution environment must be able to access the classbase of an agent. It can be either a WWW server (identified by "http://...") or another agent execution environment, i.e. a class server, (identified by "rmi://...") that also provides a mechanism for retrieving classes.

An agent needs to implement the runnable interface, i.e. a method run(). The method run() is executed whenever an agent is created or arrives at a new place. It implements the behavior of an agent.

The method move() allows an agent to change the place it is running in. It calls the method createAgent() at the new place and passes itself as the initial state. Then the local entry of the context is deleted. Since the JavaVM does not allow to continue directly after the call of move() on arrival at the new place, the method run() will be entered again from the very beginning. This is a drawback since it slightly complicates the programming of the behavior within the method run().

The usual method invocation can be used for the interaction between agents residing at the same place and thus within the same JavaVM. Remote method invocation is supported for interacting with agents residing at different places. 


\subsection{Name Server}

A simple name server is provided by Sun within the remote method invocation facility. It is the java.rmi.Registry class. The implementation allows to resolve URLs to object references. Unfortunately, it provides only a flat name space.

\subsection{Class Server}

The class ClassServer provides only the method getClass() at a remote interface. It can be used to load missing code from the originating JavaVM of an agent. Alternatively a WWW server might be installed to act as the class base.

When passing an agent object via the remote method invocation interface to a new place, all local objects referenced directly or indirectly by the agent object are copied as well. Therefore an agent can be implemented as several Java objects. The object serialization algorithm will go over the entire graph of objects and write it to a stream, so that it can be completely reconstructed on the receiving side. It preserves identity, so that two object references which are pointing to the same object will also point to the same object in the new place. Furthermore cyclic structures are detected and reconstructed accordingly.

This is not done in case of a reference to a remote object. References to remote objects form the border of an agent. In fact they are lost when we don't care about them and an agent is moving from one host to the next as we will discuss under the list of open issues below.

The execution environment at certain place can have special features which are not coded in Java and are therefore not movable to other locations. Indeed this is an approach which is followed in some agent based network management solutions. The JavaVM is placed within a management platform which provides the communication stacks for speaking SNMP and CMIP to the outside world. For the Java program there is a special API for accessing these communication stacks. Here the agent facility is limited to download new code. This seems to be a good intermediate solution until implementations of communication protocols completely coded in Java become available. They are necessary for the agents to be usable in any place. At least for SNMP there already exist two implementations as already mentioned above.

The mobile agent technology is seen as a complement to client-server computing rather than as displacement for it. First of all the remote method invocation and thus client-server computing enables the mobile agent execution environment since it is used to pass state and code of agents between different hosts. Furthermore, agents can use the remote method invocation to communicate with remote agents providing a service.

\subsection{Open Issues}

Currently object addresses are used to reference remote objects. Thus each move of an agent that offers a service at a remote interface results in open references. There is no possibility to locate the agent afterwards without additional mechanisms. One solution is to keep a forwarding entry at each place which has been visited by an agent. Unfortunately, this would result in very big tables at each host and waste a lot of computing time for resolving a reference as soon as the number of agents and hosts increases. A better solution uses object names to store references. Object names can then be resolved at the 
central naming server.

It is not possible to transparently keep client-server relations via remote interfaces alive while an agent is moving from one place to another. Note, that it does not only affect the moving agents itself but also the clients that are connected to a service offered by the moving agent. Where a mobile agent is acting as a client it can simply close down the connection to its server and re-open it connecting to the same address from the new location. Where a mobile agent is acting as a server it must close the incoming connections and free the port. At the new location it has to choose a new port where it will offer its service. Then the service of the moved agent needs to be announced under the old name with the new address at the central naming server. This enables the clients which are bound to the service to re-open there connections after resolving the service name again via the central naming server.

It is possible to model relationships between agents. They are also modeled as remote objects. Thus the simple copy operation invoked during remote method invocation will stop when encountering a reference to a relation object. In such a case a new copy operation has to be provided that takes care of the relation graph and traverses it. Whether a referenced object will be copied or not is decided based on the attributes of the relation. This approach is taken in the COSCompoundLifeCycleService of Corba [COS]. It might be helpful to have a similar service in our environment.

A very important issue is security. Currently there are security restrictions neither at the remote interface for creating agents nor at the remote interface for retrieving classes. If the environment should be opened to a public network where we have to deal with malicious agents at least authorization and authentication mechanisms are required.

Mechanisms to make code and state persistent simplify the implementation of agents. Code and state persistence is of special interest if loading an agent over the network consumes a lot of resources and thus also takes a long time. State persistence is always necessary if the state can not be gathered from other resources. A good example are accounting records which cannot be generated again. Thus a persistence service is called for.

\section{EXAMPLE SCENARIO}

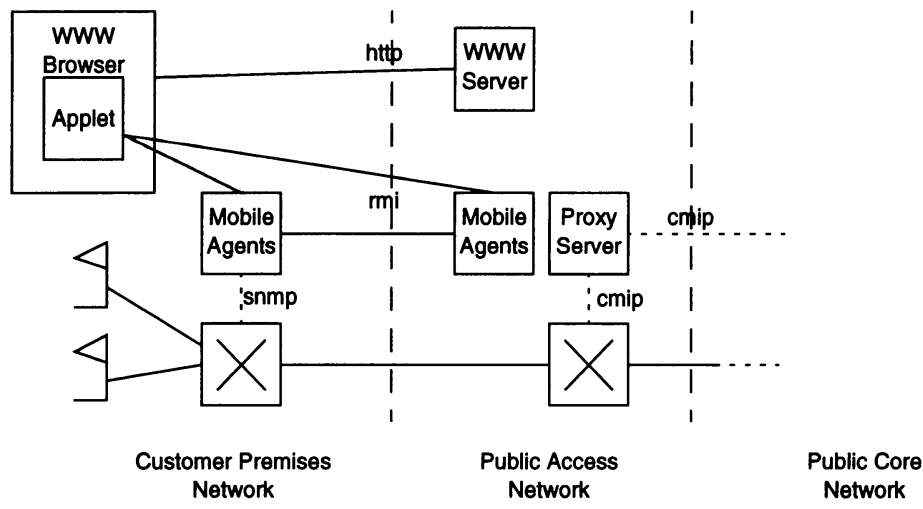

Figure 5 Example scenario. 
The connectivity service provider has set-up a WWW server as well as an agent execution environment in his domain. On the WWW server he provides freely accessible information about his service and a form for subscription. Furthermore a closed environment for configuration and supervision of the connectivity service.

In case of an outsourcing scenario the customer first subscribes to the service. Then the customer administrator has to download the basic agent execution environment. It has to be installed, at least once, in each of the customer premises networks. During configuration of the connectivity service the customer passes the addresses of these places to the service provider. This allows the service provider to send around an initial agent and discover the equipment that is installed in the customer domain. In our prototype we use ping and SNMP requests to detect hosts and ATM switches. Then initial agents for connection and IP configuration are sent to the customer domain.

As soon as the infrastructure is in place the customer gets access to WWW pages to configure and supervise its connections in its own and the public networks. He can setup and clear down ATM virtual path and virtual channel connections. In addition monitoring of cell counters can be done in real-time. The graphical user interface can be accessed from each WWW browser, wherever the customer administrator is located.

As soon as a misbehavior is detected an alarm is raised and sent to the network administrator in the service provider domain. Furthermore a trouble ticket informs the customer about the problem and the estimated time of recovery. The service provider can now use additional agents to test the equipment in the customer or its own domain and track down the fault to a specific part of the network. As long as the problem can be solved without changes of hardware modules there is no need for the network manager to send out a technician.

Note, that the scenario still includes a proxy server to access equipment in the public domain via CMIP. This is required since the network and network element level interfaces as well as the cooperative interfaces in public domains are currently standardized based on CMIP. Nevertheless, towards the customer we are in favor of a WWW and Java based solution due to its minimum requirements concerning the hardware platform and its simplicity for a customer and the service provider.

\section{RELATION TO OTHER WORK}

There are many agent systems that are currently under development not only in research projects but also in industry. Telescript by General Magic is likely the most elaborated agent environment since the requirements of mobile agents were taken into account from the very beginning of its design. For example the execution at a new place starts directly behind the move statement what greatly simplifies programming. Others include AgentTcl by Dartmouth College or ARA by the University of Kaiserslautern.

Most of the well established agent platforms provide more elaborated features for agent programming than Java. Nevertheless, there is very little support for a global usage. We have chosen Java because of its market strength and the upcoming support on nearly all computing platforms. Furthermore, it is very easy to build a basic agent environment. There are several other agent platforms based on Java currently under way, e.g., Java-to-Go by the University of Berkeley and the Aglets library by IBM Tokyo.

Another technology of interest in our field is Corba. Corba provides the base for cli- 
ent-server computing. It allows to pass the state of objects. Unfortunately it is not possible to pass code since there is no virtual machine by default but the object behavior is compiled into native code. In order to achieve code portability one might restrict the implementation environment to a specific language that is compiled to a virtual machine, like Smalltalk or Java for example. Corba might be an alternative for doing the remote method invocation but since we have a homogenous Java environment we don't need the language independent interface description and coding. Nevertheless there are many services already specified and partially implemented which are of interest also to an agent based environment, e.g., the event service. Here we can definitely benefit from Corba compatible interfaces.

\section{SUMMARY AND OUTLOOK}

We have shown how the WWW and Java can be applied in the field of broadband connectivity service provisioning. This solution puts only minimum requirements on the computing platform at the customers site since Java is light weight and portable. Software components can easily be updated or extended by a service provider. As soon as Java will be part of operating systems, as it has already been announced by many computer vendors, like Microsoft, Sun, and IBM for example, it will be available from scratch on nearly all hosts. It is foreseen that such solutions enable the forthcoming network computing technology as drawn by Oracle.

Furthermore, the usage of WWW reduces the human interaction required on behalf of the service provider. Both the customer as well as the service provider can benefit from the simplicity of WWW based interfaces. Therefore the solution is likely to reduce cost at both sites. Via the WWW a customer can easily find a service provider that meets his requirements and a service provider can reach an increasing number of (potential) customers.

The basic idea of WWW and Java based network management can be used in any area of network and service management. For example it also provides a good base for intranet management solutions that can be sold to customers. In this case both the customer and the service provider can benefit from the fact that the implementation can run on any platform with a JavaVM. Furthermore software release and update management can be simplified by setting up a server that is contacted by the application on start-up or from within a running process without an interaction of a user.

Before such a solution can be applied at a public customer-to-service provider reference point the open security issue needs to be addressed. To protect both the customer as well as the service provider side from malicious attacks through the mobile agent facility. A simple WWW based interface can already be set-up since the security requirements can normally be satisfied by a firewall.

\section{REFERENCES}

[ACCT] Greene, W., Heinanen, J., McCloghrie, K., Prasad, A.: "Managed Objects for Managing the Collection and Storage of ATM Accounting Information", Internet 
draft, IETF, April 1996, work in progress.

[ADVENT] Advent Network Management, Inc.: "Advent Network Java SNMP Package”, Version 1.0 Beta, http://www.adventnet.com/snmp_api.html, 1996.

[CORBA] OMG: "The Common Object Request Broker: Architecture and Specification", Revision 2.0, July 1995.

[COS] OMG: “CORBAservices: Common Object Services Specification”, Revision 1.0, March 1995.

[DERI] Deri, L.: "Network Management for the 90s", to be published.

[DMFA] Garcia-Lopez, E.: "Distributed Management Facilities Architecture", Version 2.0, TINA-C, March 1996.

[ISO10165-4] ISO/ITU-T: "Guidelines for the Definition of Managed Objects", ISO/ IEC 10165-4, ITU-T Recommendation X.721, 1992.

[JASCA] Nikander, P., Wessman, P.: "Java SNMP Control Applet”, http://termiitti.akumiitti.fi/nixu/, 1996.

[JOS] Sun Microsystems, Inc.: "Java Object Serialisation Specification”, Revision 0.9, May 1996.

[JRMI] Sun Microsystems, Inc.: "Java Remote Method Invocation Specification", Revision 0.9, May 1996.

[M3] ATM Forum: "Customer Network Management (CNM) for ATM Public Network Service (M3 Specification)”, Revision 1.04, October 1994.

[RFC1157] Case, J., Fedor, M., Schoffstall, M., Davin, J.: “A Simple Network Management Protocol (SNMP)”, RFC1157, IETF, May 1990.

[RFC1695] Ahmed, M., Tesink, K.: "Definitions of Managed Objects for ATM Management Version 8.0 using SMIv2”, RFC1695, IETF, August 1994.

[WEST] Westerkamp, E.: “Automatic Software Distribution of Java Applications".

[WREG] Wreggit, D. J.: "Software Agents Using Java”, December 1995.

[XOJIDM] X/Open Company Ltd.: "Inter-Domain Management Specifications: Specification Translation”, Second Sanity Check Draft, June 1996, work in progress. 